rim surrounding the lesion in $92 \%$ of patients. This paper is very important for calling the attention of the radiologist and urologist in order to recognize segmental testicular infarction and thus to avoid unnecessary orchiectomy. The authors concluded that these imaging findings (ultrasound and complimentary MRI in difficult cases), associated with the negative tumoral markers and short follow-up, should allow confidence in the diagnosis and thus avoid orchiectomy.

Dr. Adilson Prando

Chief, Department of Radiology

Vera Cruz Hospital

Campinas, São Paulo, Brazil

\title{
UROGENITAL TRAUMA
}

\author{
The treatment of posterior urethral disruption associated with pelvic fractures: comparative \\ experience of early realignment versus delayed urethroplasty \\ Mouraviev VB, Coburn M, Santucci RA \\ Prostate Centre, Vancouver General Hospital, Vancouver, British Columbia, Canada \\ J Urol. 2005;173: 873-6
}

Purpose: Urological treatment of the patient with severe mechanical trauma and urethral disruption remains controversial. Debate continues regarding the advisability of early realignment vs delayed open urethroplasty. We analyzed our experience with 96 patients to determine the long-term results of the 2 approaches.

Materials and Methods: We retrospectively reviewed the records of 191 men with posterior urethral disruption after severe blunt pelvic injury between 1984 and 2001, of whom 96 survived. Data on 57 patients who underwent early realignment were compared to those on 39 treated with delayed urethroplasty with an average 8.8-year followup (range 1 to 22). All patients were evaluated postoperatively for incontinence, impotence and urethral strictures.

Results: The majority of patients had severe concomitant organ injuries (78\%) and severe pelvic fractures $(76 \%)$. The overall mortality rate was $51 \%$. Diagnosis of urethral rupture was based on clinical findings and retrograde urethrography. Strictures developed in $49 \%$ of the early realignment group and in $100 \%$ of the suprapubic tube group. Impotence $(33.6 \%)$ and incontinence $(17.7 \%)$ were less frequent in the early realignment group than in the delayed reconstruction group ( $42.1 \%$ and $24.9 \%$, respectively). Patients with delayed reconstruction underwent an average of 3.1 procedures compared with an average of 1.6 in the early realignment group.

Conclusions: Early realignment may provide better outcomes than delayed open urethroplasty after posterior urethral disruption. Increased complications are not seen and, although it can be inconvenient in the massively injured patient, it appears to be a worthwhile maneuver.

\section{Editorial Comment}

Mouraviev et al, detail their extensive experience with a retrospective review of 191 urethral disruption injuries. The acute management of pelvic fracture and associated urethral injury is controversial. Classically, acute management is a "delayed approach" of placement of a suprapubic tube, percutaneously, if the bladder is 
full, or open, if the bladder is decompressed or has a concomitant bladder or bladder neck injury. After a minimum period of three months, the urethral injury is reconstructed. This method of delayed definitive management is particularly useful in rural or small hospitals where no Urologist is available. The main complication of this method is the near $100 \%$ stricture rate. Of historical interest is open primary repair of acute urethral disruption, which is absolutely contraindicated due to technical difficulty, risk of uncontrolled pelvic bleeding, and unacceptably high rates of impotence and incontinence.

The other management option that has gained considerable recent support is delayed or immediate primary urethral realignment by endoscopic means. Here, two cystoscopes, one antegrade and one retrograde, are used to get a wire and catheter across the urethra. The immediate approach is reserved for the stable patient with a short urethral distraction distance. The delayed approach is a type of damage control, where the unstable patient is first resuscitated and then when stable, the urethra is later realigned (usually after 2 to 10 days, typically concomitantly when Orthopedics internally fixes the pelvic fracture).

Contrary to prior reports, the authors here report higher impotence (42\%) and incontinence (25\%) with the delayed approach over early realignment. This is difficult to explain since such complications are thought to be from the mechanism of the original injury. Regardless, similar to the literature, primary realignment appears here to reduce the eventual stricture and impotence rates. When posterior strictures do occur, it appears that the eventual stricture may be shorter and easier to manage. This may be a selection bias, since patients with the most severe urethral distractions are typically the most severely injured and unstable, and thus often not primarily realigned. Another added benefit to realigning the urethra is that the suprapubic tube can be removed and not be in the way of any inguinal orthopedic incisions. Primary urethral realignment makes good common sense, appears to reduce complications; and is thus an integral management tool in the contemporary management of urethral distraction injuries.

Dr. Steven B. Brandes

Associate Professor, Division of Urologic Surgery Washington University in St. Louis

St. Louis, Missouri, USA

\section{Traumatic ureteral injuries: a single institution experience validating the American Association for the Surgery of Trauma-Organ Injury Scale grading scale \\ Best CD, Petrone P, Buscarini M, Demiray S, Kuncir E, Kimbrell B, Asensio JA \\ Department of Urology and Division of Trauma and Critical Care, Los Angeles County and University of Southern California, Los Angeles, California, USA \\ J Urol. 2005; 173: 1202-5}

Purpose: Ureteral injuries are uncommon and challenging. In this study we report our institutional experience with ureteral injuries. We evaluated the American Association for the Surgery of Trauma-Organ Injury Scale (AAST-OIS) for ureteral injuries as a predictor of outcomes for complexity of repair, morbidity, mortality and associated injuries.

Materials and Methods: We performed a retrospective, 120-month study (January 1992 to December 2002) at an urban, level I trauma center.

Results: In the 57 patients mean hospital Admission blood pressure +/- SD was 115 +/- $25 \mathrm{~mm} \mathrm{Hg}$, mean Revised Trauma Score was 7.38 +/- 0.84 and mean Injury Severity Score was $15+/-1.15$. The mechanism of injury was penetrating in 55 cases $(96.5 \%)$, including gunshot wound in $52(54.5 \%)$ and stab wound in 2 
(5.5\%), and in blunt 2 of motor vehicle accidents (3.5\%). The anatomical location was the left side in 33 cases $(58 \%)$, right side in $23(40 \%)$ and bilateral in $1(2 \%)$. The distribution of injuries was proximal in 15 cases (26\%), mid in $21(37 \%)$ and distal in $21(37 \%)$. Associated injuries were present in 56 patients (98\%). An intraoperative diagnosis was made in 44 cases $(77 \%)$. Of the patients $50(88 \%)$ required complex repairs or an adjunct procedure, including a double pigtail stent in $33(58 \%)$, ureteroureterostomy in $20(35 \%)$, ureteroneocystostomy with a psoas hitch in $10(18 \%)$, external diversion in $9(16 \%)$, suprapubic cystostomy in $8(14 \%)$, nephrostomy in $2(3.5 \%)$, nephrectomy in $2(3.5 \%)$ and ligation in $2(3.5 \%)$. Injury grade was I to V in $5(8 \%), 8(13 \%), 13(22.8 \%), 18(31.6 \%)$ and $13(22.8 \%)$ cases, respectively. Overall 51 patients $(89 \%)$ survived. No deaths were related to ureteral injury. Renal salvage was achieved in 49 of the 51 surviving patients $(96 \%)$.

Conclusions: Ureteral injuries are uncommon. The complexity of repair and number of associated injuries increase with AAST-OIS injury grade. Mortality increases with AAST-OIS injury grade but it is not related to the ureteral injury. Excellent results can be achieved with complex techniques of primary repair, leading to renal salvage.

\section{Editorial Comment}

The American Association for the Surgery of Trauma developed an organ injury scale for each of the Urologic organs, as published in the Journal of Trauma in 1989 and 1992 (1,2). The great value in such scales is that they enable a uniform method of defining urologic injuries and so facilitate the development of comparative studies and research. These injury scales were based on the consensus of experts in urologic trauma, and not by evidence based medicine. The above retrospective review is another in a long line of papers seeking to validate that the complexity of repair and outcomes correlate to the AAST scale of degree of injury.

Other lessons learned from this study are that ureteral injuries are rare events. The majority of ureteral injuries are due to penetrating trauma. The location of penetrating ureteral injuries is roughly evenly distributed among each one-third of ureter. Penetrating injuries have a very high associated organ injury rate, and it is the associated injuries that primarily cause the morbidity and potential mortality. The most reliable method to diagnosing a ureteral injury is intraoperative direct exploration. Ureteral injuries from blunt trauma are typically due to deceleration and at the ureteropelvic junction.

\section{References}

1. Moore EE, Cogbill TH, Jurkovich GJ, McAninch JW, Champion HR, Gennarelli TA, Malangoni MA, Shackford SR, Trafton PG: Organ injury scaling. III: Chest wall, abdominal vascular, ureter, bladder, and urethra. J Trauma. 1992; 33 : 337-9.

2. Moore EE, Shackford SR, Pachter HL, McAninch JW, Browner BD, Champion HR, Flint LM, Gennarelli TA, Malangoni MA, Ramenofsky ML, et al.: Organ injury scaling: spleen, liver, and kidney. J Trauma. 1989; 29: 1664-6.

Dr. Steven B. Brandes Associate Professor, Division of Urologic Surgery Washington University in St. Louis St. Louis, Missouri, USA 